\title{
The fate of the distal aorta after repair of acute type $A$ aortic dissection
}

\author{
James C. Halstead, MA (Cantab), MB, BChir, MRCS (Eng), ${ }^{a}$ Matthias Meier, MD, ${ }^{a}$ Christian Etz, MD, ${ }^{a}$ \\ David Spielvogel, MD, ${ }^{a}$ Carol Bodian, DrPH, ${ }^{b}$ Michael Wurm, MD, ${ }^{a}$ Rohit Shahani, MD, and Randall B. Griepp, MD ${ }^{a}$
}

Supplemental material is available online.
From the Departments of Cardiothoracic Surgery $^{\mathrm{a}}$ and Anesthesiology, ${ }^{\mathrm{b}}$ Mount Sinai School of Medicine, New York, NY.

Read at the Eighty-fifth Annual Meeting of The American Association for Thoracic Surgery, San Francisco, Calif, April 10-13, 2005.

Received for publication April 12, 2005; revisions received June 15, 2006; accepted for publication July 17, 2006.

Address for reprints: James C. Halstead, MA (Cantab), MB, BChir, MRCS (Eng), Department of Cardiothoracic Surgery, Mount Sinai School of Medicine, One Gustave L. Levy Place, New York, NY 10029 (E-mail: jameschalstead@yahoo.co.uk).

J Thorac Cardiovasc Surg 2007;133:127-35

$0022-5223 / \$ 32.00$

Copyright (C 2007 by The American Association for Thoracic Surgery

doi:10.1016/j.jtcvs.2006.07.043
Objectives: The residual aorta's behavior after repair of acute type A dissection is incompletely understood. We analyzed segmental growth rates, distal reoperation, and factors influencing long-term survival.

Methods: One hundred seventy-nine consecutive patients (70\% male; mean age, 60 years) with acute type A dissection underwent aggressive resection of the intimal tear and open distal anastomosis (1986-2003). Hospital mortality was 13.4\%. Survivors had serial computed tomographic scans: digitization yielded distal segmental dimensions. Segment-specific average rates of enlargement and factors influencing faster growth were analyzed. Distal reoperations and patient survival were examined.

Results: Eighty-nine (57\%) patients had imaging data sufficient for growth rate calculations. The median diameters after repair were as follows: aortic arch, $3.6 \mathrm{~cm}$; descending aorta, $3.7 \mathrm{~cm}$; and abdominal aorta, $3.2 \mathrm{~cm}$. Subsequent growth rates were $0.8,1.0$, and $0.8 \mathrm{~mm} / \mathrm{y}$, respectively. Initial size of greater than $4 \mathrm{~cm}(P=$ $.005)$ and initial diameter of less than $4 \mathrm{~cm}$ with a patent false lumen $(P=.004)$ predicted greater growth in the descending aorta, and male sex $(P=.05)$ significantly affected growth in the abdominal aorta. No significant factors were found for the aortic arch. There were 25 distal aortic reoperations (16 patients), and risk of reoperation was $16 \%$ at 10 years. Risk factors reducing long-term survival after repair of acute type A dissection included age $(P<.0001)$, new neurological deficit at presentation $(P=.04)$, absence of preoperative thrombus in the false lumen of the ascending aorta $(P=.03)$, and a patent distal false lumen postoperatively $(P=.06)$ but not distal reoperation.

Conclusions: Growth of the distal aorta after repair of acute type A dissection is typically slow and linear. Distal reoperation is uncommon, and late risk of death is approximately twice that of a healthy population.

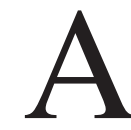
lthough acute type A aortic dissection remains a challenging condition to manage, the results from emergency surgical treatment are continuing to improve as the result of advances in diagnosis, as well as better surgical techniques and perioperative care. Unfortunately, surviving the initial course does not guarantee freedom from subsequent aortic events because much of the thoracoabdominal aorta frequently remains dissected and at risk of aneurysm formation. However, there is a paucity of data regarding the long-term behavior of the distal aorta after type A dissection. This study was undertaken to assess the long-term outcomes after acute type A dissection, especially with regard to distal aortic segmental growth rates, distal aortic reoperations, and survival.

\section{Patients and Methods}

Patient Data Abstraction

The aortic surgery database at Mount Sinai School of Medicine contains 179 consecutive patients who underwent emergency operations for acute type A dissection between January 


$$
\begin{aligned}
& \text { Abbreviations and Acronyms } \\
& \begin{aligned}
\text { CAT } & =\text { computed axial tomography } \\
\text { COPD } & =\text { chronic obstructive pulmonary disease } \\
\mathrm{CT} & =\text { computed tomography } \\
\mathrm{HR} & =\text { hazard ratio } \\
\mathrm{OR} & =\text { odds ratio }
\end{aligned}
\end{aligned}
$$

1986 and April 2003. The patients' clinical characteristics are shown in Table 1. The diagnosis of acute type A dissection was made on the basis of preoperative imaging (computed tomography [CT], magnetic resonance imaging, aortography, or echocardiography) or at the time of the operation. The dissection characteristics (including transesophageal echocardiographic assessment of flow patterns), extent of distal resection, and technique of proximal reconstruction are shown in Table 2.

\section{Operative Procedures}

Although surgical techniques evolved somewhat over the time course of this series, the basic principles involved replacement of the ascending aorta and resection of the primary intimal tear, with the construction of an open distal anastomosis during a period of hypothermic circulatory arrest. The site of arterial cannulation changed with time, with preferential use of the right axillary artery over the femoral artery in recent years. In all patients, the flow pattern in the descending aorta was carefully monitored by means of transesophageal echocardiography at the start of perfusion to avoid malperfusion. During cooling, the aorta and heart were not manipulated until ventricular fibrillation occurred. The ascending aorta was then crossclamped in its distal half, and the aorta was opened. Cardioplegia was delivered directly into the coronary ostia, and the aortic pathology was assessed.

The extent of operation was determined by each of several surgeons. However, composite graft replacement was used almost exclusively when the coronary sinuses were dissected. This aggressive strategy of resection rather than repair of the dissected aortic root resulted in a high proportion of composite graft replacements. ${ }^{1}$

When conservative repair was undertaken, the aorta was transected at the sinotubular junction and reinforced with an externally placed circumferential piece of Teflon felt (DuPont, Parkersburg, WV) and an internal gusset of felt or autologous pericardium, so as to realign the commissural geometry. Tissue glues were not used. Occasionally, separate valve replacement was undertaken in cases of bicuspid, stenotic, or residually regurgitant valves.

For the composite graft replacements, a manufactured conduit with a mechanical valve was used in most patients. In a minority of patients, a biologic valve was sutured to an appropriately sized tubular woven graft. Coronary reconstruction was usually undertaken by using buttons on the outside of the graft (the buttonBentall technique).

After the proximal repair, during a single period of hypothermic circulatory arrest, the arch was inspected, and an open distal anastomosis was undertaken. The presence and site of the intimal tear or fragile tissues in the arch determined the extent of distal
TABLE 1. Patients' clinical characteristics

\begin{tabular}{lc}
\hline Factor & $\begin{array}{c}\text { No. (\%), except } \\
\text { where indicated }\end{array}$ \\
\hline Age (mean \pm SD) & $60.4 \pm 14.5$ \\
Male sex & $125(70)$ \\
Coronary artery disease & $32(18)$ \\
Chronic pulmonary disease & $11(6)$ \\
Smoking & $91(51)$ \\
Diabetes & $12(7)$ \\
Hypertension & $139(78)$ \\
Renal failure & $5(3)$ \\
Marfan syndrome (or forme fruste) & $15(8)$ \\
Prior sternotomy & $20(11)$ \\
Prior neurological event & $14(8)$ \\
New neurological deficit at presentation* & $27(15)$ \\
Hemodynamic compromise & $32(18)$ \\
Malperfusion syndrome at presentationt & $24(13)$ \\
\hline
\end{tabular}

*Transient ischemic attack or acute cerebrovascular accident at the time of presentation. †Absent peripheral pulse with appropriate symptomatology to indicate an acute loss or absence of renal opacificaton on computed tomographic scan.

resection (Table 2). The dissected layers were reapproximated with supporting external Teflon felt. The distal end of the graft was sewn within this construct, and the arch or arch graft was carefully deaired. Antegrade reperfusion was instituted through an arterial cannula inserted into the ascending graft in cases in which cardiopulmonary bypass had been initiated through the femoral artery; otherwise, perfusion was resumed through the right axillary artery.

\section{Follow-up}

Surviving patients were invited for annual clinical review and CT scanning of the entire aorta. Clinical data were compiled from these office visits, telephone contact with patients, and input from primary care physicians. It included, but was not limited to, information concerning aortic reoperations or ruptures, thromboembolic events, hemorrhagic complications, cardiac pathology (eg, episodes of endocarditis), and patient survival. Cause of death, where available, was also recorded. The median patient follow-up time after hospital discharge was 61 months (range, 0-218 months).

\section{Growth Rates}

A process of CT digitization, previously described in detail, ${ }^{2}$ was used to assess the whole of the residual aorta after surgical intervention. Segmental expansion rates were calculated by using dimensions derived from multiple CT scans. For inclusion into analysis of expansion rates, at least $2 \mathrm{CT}$ scans had to have been taken postoperatively at least 6 months apart. Once an aortic segment had been subject to reoperative surgical intervention, its dimensions were no longer considered part of the growth rate calculations. CT scans were also analyzed for evidence of a patent false lumen.

A number of patients died before 2 postoperative studies at least 6 months apart could be completed, others declined further studies, and a few were lost to follow-up. Thus only $89(57 \%)$ 
TABLE 2. Dissection characteristics, including extent of distal resection and method of proximal reconstruction

\begin{tabular}{|c|c|}
\hline & No. $(\%)$ \\
\hline \multicolumn{2}{|l|}{ Dissection characteristics } \\
\hline Preoperative false lumen thrombosis* & $33(18)$ \\
\hline Free blood in the pericardium & $62(35)$ \\
\hline Contained aortic hematoma & $74(41)$ \\
\hline \multirow{2}{*}{ Operative appearance of the ascending aortic false lument } & Patent: 162 (91) \\
\hline & Thrombosed: 17 (9) \\
\hline \multirow[t]{3}{*}{ Operative appearance of the aortic arch false lument } & Patent: 149 (83) \\
\hline & Thrombosed: 21 (12) \\
\hline & None seen: $9(5)$ \\
\hline \multirow[t]{3}{*}{ Operative appearance of the descending aortic false lument } & Patent: 137 (77) \\
\hline & Thrombosed: 12 (7) \\
\hline & None seen: 30 (17) \\
\hline Tear located in the ascending aorta & $128(72)$ \\
\hline Tear located in the aortic arch & $32(18)$ \\
\hline Tear located in the descending aorta & $4(2)$ \\
\hline Tear located in the ascending aorta and aortic arch & $8(4)$ \\
\hline Tear located in the aortic arch and descending aorta & $4(2)$ \\
\hline Tears located in the ascending and descending aorta & $1(1)$ \\
\hline No tear found & $2(1)$ \\
\hline \multicolumn{2}{|l|}{ Distal replacement } \\
\hline Ascending aorta only & $65(36)$ \\
\hline Ascending aorta and hemiarch & $97(54)$ \\
\hline Ascending aorta and total arch & $11(6)$ \\
\hline Ascending arch and upper descending aorta & $6(3)$ \\
\hline \multicolumn{2}{|l|}{ Proximal reconstruction } \\
\hline Valve resuspension & $97(54)$ \\
\hline Valve repair & $2(1)$ \\
\hline Separate valve replacement & $5(3)$ \\
\hline Valve repair with sinus excision (method of Yacoub) & $1(1)$ \\
\hline Mechanical composite root replacement & $63(35)$ \\
\hline Biologic composite root replacement & $11(6)$ \\
\hline
\end{tabular}

*Either no flow pattern was observed in the ascending aortic false lumen on echocardiography (usually transthoracic) or no contrast enhancement was seen within the false lumen on computed tomographic scanning. $\dagger$ By definition, all cases have an ascending aortic false lumen, which is either thrombosed or patent at the time of surgical intervention; with the arch and upper descending aorta, these 2 possibilities exist, or the aorta can be intact and nondissected.

patients were included in the analyses for CT-derived rates of expansion. A number of factors were compared between patients who contributed CT scans and those who did not to determine whether the group who contributed scans was representative of the entire cohort of patients who survived the procedure. The factors examined were as follows: age; sex; presence of aortic dilatation, chronic obstructive pulmonary disease (COPD), diabetes, or Marfan syndrome; a history of hypertension or diabetes; the presence of thrombosis in the false lumen of the ascending aorta, hemodynamic compromise, or new neurological symptoms at presentation; use of a composite graft at the time of the operation; postoperative descending aortic dilatation; or the presence of an open false lumen.

\section{Statistical Methods}

Hospital death was defined as death within the same hospitalization. From a wide array of potentially important clinical and dissection-related variables, independent risk factors for hospital death were identified by means of logistic regression analysis. Similarly, the influence of these factors together with others reflecting the postoperative course, such as postoperative patency of aortic false lumina and reoperations, on late survival was examined with Cox proportional hazards regression. Late actuarial survival and risk factors for death were assessed among hospital survivors. For the purposes of comparison with other published data, overall actuarial survival from hospital admission is also given. Survival rates for a cohort matched for age and sex to the hospital survivors were derived from data from the 1990 Vital Statistics of the United States.

The growth rates of the serial CT scans of patients contributing 2 or more scans at least 6 months apart are described in 2 ways. First, the observed measurements of the serial scans were shown in "spaghetti plots" for each segment. Then the slopes of the rates of growth in each patient for each segment were estimated separately, and the medians of these estimated slopes are given. Finally, data 
from the serial CT scans were analyzed by using hierarchical linear models for repeated measures to study factors that influenced the growth rates.

Reoperations to resect segments of the residual native aorta were classified according to site: arch, descending aorta, thoracoabdominal aorta, abdominal aorta, or aortic root. Cumulative incidence functions ${ }^{3,4}$ were calculated to estimate the rates of reoperation distal to the prior resection at 1,5 , and 10 years.

Statistical analyses were performed with SAS software (Version 8; SAS Institute, Inc, Cary NC).

\section{Results}

\section{Hospital Death}

The overall hospital mortality rate was $13.4 \%$ (24/179). The causes of hospital mortality are cited in Table 3. Logistic regression analysis showed chronic pulmonary disease (COPD; odds ratio $[\mathrm{OR}], 13.1 ; P=.002)$, diabetes (OR, 5.8; $P=.03$ ), malperfusion (OR, 14.3; $P<.0001$ ), and hemodynamic compromise (OR, $4.5 ; P=.008)$ as independent risk factors for hospital death. The Hosmer-Lemeshow goodness-of fit-statistic (c) for this model was 0.86 .

\section{Segmental Growth Rates in the Distal Aorta}

Of the 155 hospital survivors, $89(57 \%)$ had at least 2 postoperative CT scans taken at least 6 months apart and were therefore amenable to analysis of growth rates. Of the 66 hospital survivors not contributing, 22 died during followup. Only 2 of these deaths were due to aortic events (distal rupture in both cases); 14 occurred before 200 days, and therefore these patients did not remain alive long enough to undergo the 2 adequately spaced scans necessary to contribute data. Of the 44 patients who did not have confirmed deaths, only 17 were truly lost to follow-up. The remaining 27 were alive at the time of study completion (confirmed by telephone contact or their having attended for clinic review).

Two significant differences were found between the patients contributing scans and those who did not: a higher proportion of patients contributing scans had preoperative false luminal thrombosis in the ascending aorta $(P=.03)$, and fewer had an initial postoperative diameter in the descending aorta of $4 \mathrm{~cm}$ or larger $(P=.04)$. Because these differences might have led to an underestimation of the overall median growth rates from our sample, we show the data separately according to these factors in Table E1, which is a summary of segmental growth rates.

Of the 155 discharged patients, the status of the false lumen could be ascertained in 151, and it is noteworthy that the false lumen was patent in $66(43 \%)$. Among the patients contributing growth rates, an equivalent proportion (39/89 [44\%]) had a patent false lumen on initial postoperative examination.

Postoperative growth rates in the residual ascending aorta were not analyzed because only a few patients had residual ascending segments identified on the initial post-
TABLE 3. Causes of hospital death and late mortality

\begin{tabular}{lcc}
\hline Primary cause of death & $\begin{array}{c}\text { Hospital mortality } \\
(\mathbf{n}=\mathbf{2 4}), \mathbf{n}(\%)\end{array}$ & $\begin{array}{c}\text { Late mortality } \\
(\mathbf{n}=\mathbf{4 3}), \mathbf{n}(\%)\end{array}$ \\
\hline Cardiac* & $6(25)$ & $11(26)$ \\
Proximal aortic & $2(8)$ & 0 \\
rupture & & \\
Distal aortic rupturet & $4(17)$ & $5(12)$ \\
Neurologicalf & $4(17)$ & $4(9)$ \\
Multiorgan failure & $8(33)$ & 0 \\
Other§ & 0 & $11(26)$ \\
Infected graft & 0 & $4(9)$ \\
Unknown & 0 & $8(19)$ \\
\hline
\end{tabular}

*Almost exclusively acute ischemic phenomena, either manifest as perioperative ventricular dysfunction or late myocardial infarction. $\dagger 0 f$ the 11 patients dying from aortic rupture, only 1 survived long enough to undergo reoperation. \$These were either massive global infarctions or strokes occurring perioperatively or late strokes. §Most commonly neoplasia.

operative CT scan. We report instead a comparison of the diameter of the implanted graft, according to the manufacturer, with its diameter on the first postoperative CT scan. This analysis shows a median increase of $12.3 \%$ postoperatively in the diameter of the implanted graft.

The median maximal diameter of the aortic arch after repair was $3.6 \mathrm{~cm}$, and the subsequent median growth rate was $0.8 \mathrm{~mm} / \mathrm{y}$. Male sex was predictive of slightly higher growth rates, but this did not reach statistical significance $(P=.10)$.

For the descending aorta, the median maximal diameter was $3.7 \mathrm{~cm}$, and the median growth rate was $1.0 \mathrm{~mm} / \mathrm{y}$. An initial dimension of $4 \mathrm{~cm}$ or larger $(P=.005)$ predicted more rapid growth (median, $1.3 \mathrm{vs} 0.9 \mathrm{~mm} / \mathrm{y}$ ). In patients with an initial diameter of less than $4 \mathrm{~cm}$, a patent false lumen $(P=.004)$ was associated with faster subsequent expansion (median, 1.2 vs $0.8 \mathrm{~mm} / \mathrm{y}$ ).

The results for the abdominal aorta showed a median initial diameter of $3.2 \mathrm{~cm}$ and a median growth rate of 0.8 $\mathrm{mm} / \mathrm{y}$. Male sex was predictive of more rapid aortic expansion $(P=.05)$

Analysis of spaghetti plots, which show the behavior of the individual patients' aortic segments over time, illustrates that the growth rates in all segments are typically slow and linear. This can be seen for the aortic arch, the descending aorta, and the abdominal aorta in Figure 1.

\section{Reoperations on the Distal Aorta}

Over the time course of this series (17 years), there were 25 reoperations occurring in 16 patients affecting the residual native aorta distal to the level of initial repair. The aortic segments replaced at these reoperations, shown in Figure E1, reveal that surgical intervention was most frequently required in the aortic arch and abdominal aorta. The principal criteria for advocating reoperation were aneurysm size 

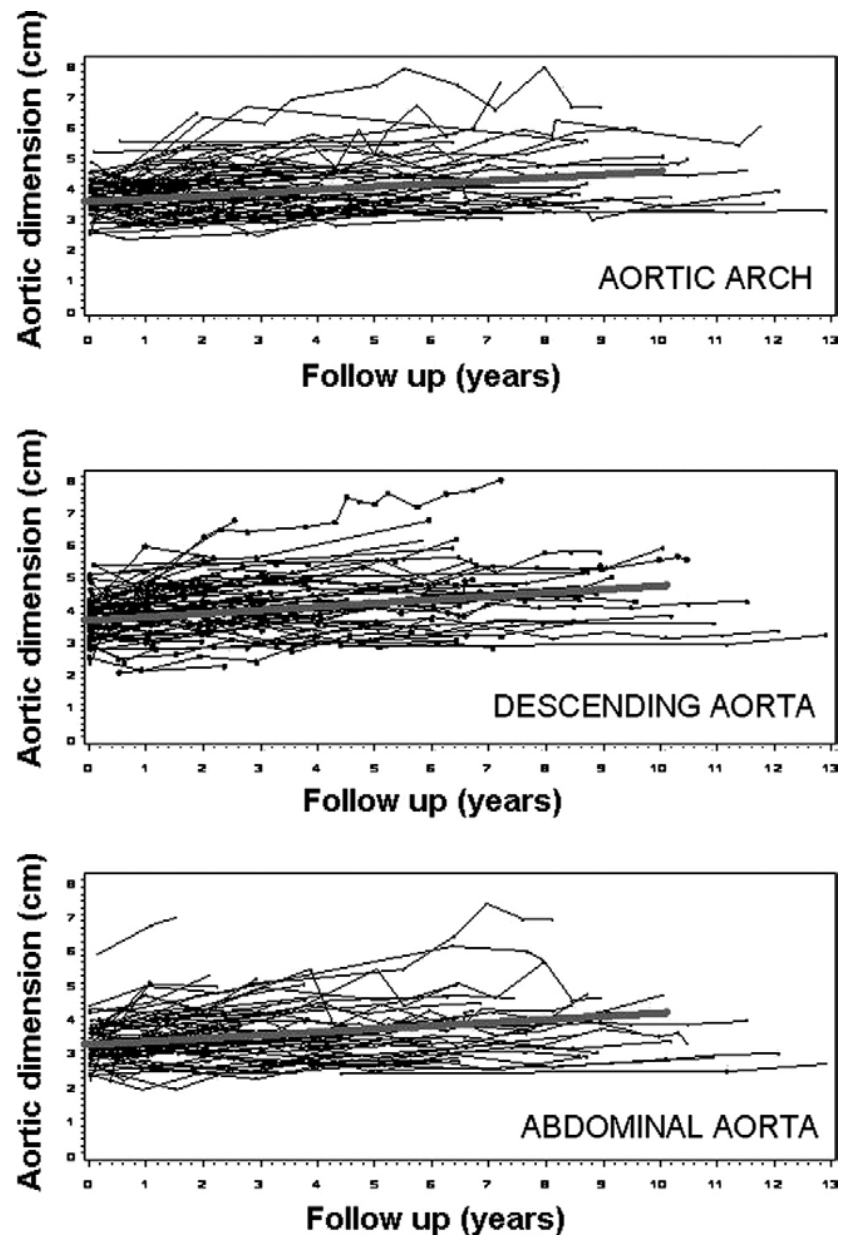

Figure 1. Individual patients' growth data for the aortic arch, descending aorta, and abdominal aorta, respectively. The individual plots end if either distal reoperation affecting that segment occurs or patient follow-up ends (death or last follow-up). The gray lines indicate the median growth rates.

or patient symptoms. The decision to reoperate in an asymptomatic patient was often determined by using a previously published equation predicting risk of rupture, ${ }^{5}$ balancing this risk against an estimate of the operative risk for a given individual. If operative risk was deemed smaller than the likelihood of rupture within a year, surgical intervention was usually advised.

Four $(31 \%)$ of the 13 discharged patients with Marfan syndrome required at least 1 reoperation in contrast with reoperations in only 12 (8\%) of 142 patients without Marfan syndrome.

There was 1 (4\%) perioperative death among these reoperations: it occurred in the only patient undergoing reoperation because of rupture (Table 3 ). The cumulative probability functions for distal reoperation at 1, 5, and 10 years are shown in Figure E1.

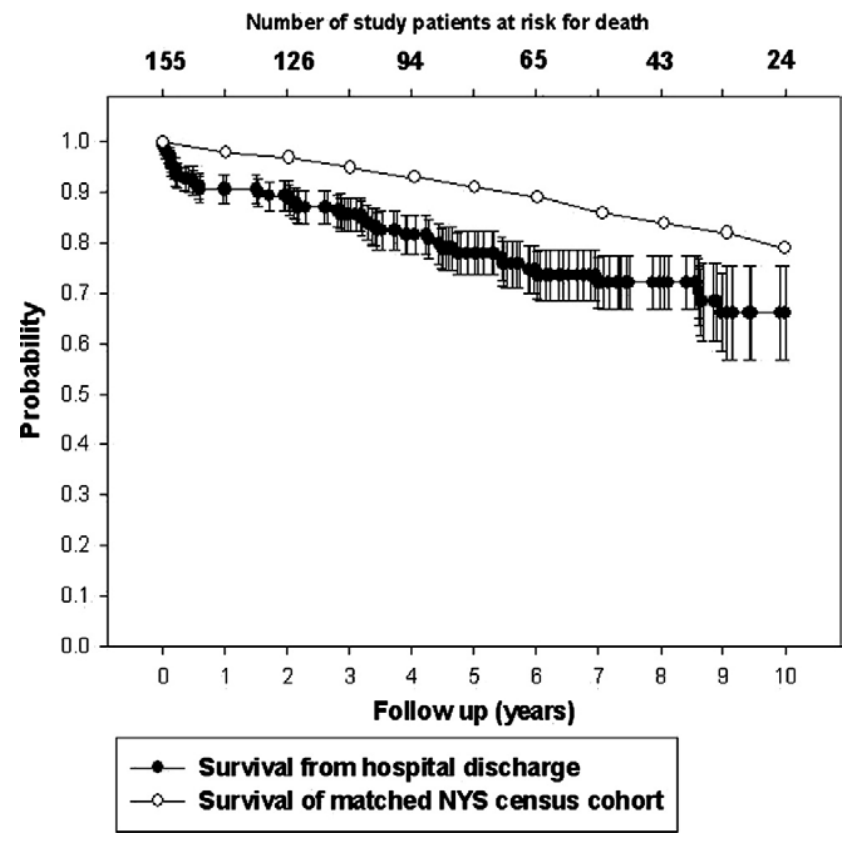

Figure 2. Late survival for acute type A surgical patients after hospital discharge and the survival of an age- and sex-matched population derived from the United States Vital Statistics (1990).

There were also 5 proximal reoperations in 5 patients for aortic regurgitation caused by the development of an aortic root aneurysm after repair.

\section{Long-term Survival}

Actuarial survival rates for hospital survivors at 1, 5, and 10 years were $90.7 \% \pm 2.3 \%, 77.9 \% \pm 3.6 \%$, and $66.2 \% \pm$ $5.1 \%$, respectively (Figure 2, seen together with the survival figures of a matched US population cohort). Cox proportional hazards regression showed that age (hazard ratio [HR], 1.1/y; $P<.0001)$, new neurological symptoms at presentation (HR, $2.3 ; P=.04$ ), and absence of preoperative false lumen thrombosis in the ascending aorta (HR, 3.8; $P=.03$ ) were independent predictors of death after hospital discharge; the presence of a patent false lumen postoperatively came close to statistical significance (HR, 1.9; $P=$ .06). Distal reoperation, entered into the model as a timechanging covariate, does not affect survival.

The causes of the 43 deaths that occurred during follow-up are listed in Table 3. It should be noted that despite efforts to ensure close surveillance, $12 \%$ of patients died of rupture, $9 \%$ of graft infection, and $19 \%$ of unknown causes, a category that likely includes additional instances of rupture.

Overall actuarial survival rates from hospital admission at 1,5 , and 10 years were $79.1 \% \pm 3.1 \%, 67.4 \% \pm 3.7 \%$, and $57.1 \% \pm 4.7 \%$, respectively. 


\section{Discussion}

\section{Hospital Mortality}

Despite improvements of all sorts in the recent care of patients with acute type A dissection, the perioperative mortality rate remains between $10 \%$ and $30 \% .^{6-15} \mathrm{We}$ identified COPD, diabetes, malperfusion, and hemodynamic compromise as key determinants of perioperative mortality, which is also in broad agreement with factors cited in previous reports of acute type A dissection: in particular, we and others have found no independent influence of age. ${ }^{16}$ Unfortunately, the factors determining hospital survival are generally not subject to modification. Thus these analyses are only useful in identifying particularly high-risk groups, in whom it can and has been argued that surgical intervention for acute type A dissection should perhaps not be undertaken. ${ }^{17}$

\section{Segmental Aortic Growth Rates}

We have shown that the median diameters of the aortic arch, descending thoracic aorta, and abdominal aorta are all mildly enlarged after type A dissection repair. The median expansion rates of these segments are typically low, approximately $1 \mathrm{~mm} / \mathrm{y}$. Unfortunately, this analysis is based on incomplete CT follow-up, which obviously limits the strength of the findings. We did compare the characteristics of the group whose scans permitted analysis of growth rates with those of the entire cohort and found they matched closely; perhaps this suggests that our analysis is relevant to patients with acute type A dissection as a whole, but of this we have no certainty. Moreover, the fact that the analyzed group included a significantly greater proportion of patients with a preoperatively thrombosed false lumen in the ascending aorta, which is associated with greater longevity but no difference in segmental growth rates, and with a significantly smaller initial postoperative diameter in the descending aorta, which does predict a slower expansion rate in that segment of the aorta, suggests that our growth rates are likely to be somewhat conservative.

The analysis of risk factors for more rapid expansion in each distal aortic segment after acute type A dissection repair has shown that male sex seems to be important in determining faster growth both in the aortic arch and abdominal aorta. In the descending aorta initial size and patency of the false lumen are the dominant factors accelerating aortic expansion: patency of the false lumen has a highly significant effect on growth rates in patients with a small initial postoperative aortic diameter.

The finding that there is an increase in diameter of the implanted graft postoperatively is not widely recognized but is consistent with our clinical impressions from the small number of patients who require reoperation in the proximal aorta.
The influence of male sex on expansion rates of the aorta is a new observation that is somewhat at variance with the evidence in a recent study by Sueyoshi and colleagues ${ }^{18}$ indicating that female sex is associated with a tendency toward more rapid expansion of the aorta after type B dissection. The faster growth of the infrarenal aorta in male subjects might be related to whatever factors cause a higher incidence of infrarenal aneurysms in male subjects without dissection.

In contrast, the influence of a patent false lumen in determining faster subsequent growth of a dissected aorta has been noted previously. ${ }^{18,19}$ Sueyoshi and colleagues ${ }^{18}$ assessed expansion rates after nonoperatively managed type $\mathrm{B}$ aortic dissection using CT scanning and found the presence of blood flow in the false lumen to be the only significant risk factor for faster growth. The mean initial segmental dimensions found were similar to those in the present study, as was the overall abdominal aortic expansion rate, but growth rates in the aortic arch and descending aorta were twice those seen in our study. In addition to methodological differences, the discrepancy in the growth rates found in these studies might be explicable on the basis of differing underlying pathology (type A vs type B dissection), sites of intimal tear, and (operative vs nonoperative) management.

Fattori and associates ${ }^{19}$ followed very closely a cohort of 70 patients after surgical intervention for acute type A dissection with serial magnetic resonance imaging. In accord with the present study, they found the yearly aortic growth rate to be maximal in the descending aortic segment and to be significantly higher in the absence of false luminal thrombosis. The median descending aortic growth rate in our study, however, is considerably less than what was found by Fattori and associates (1 vs $3.7 \mathrm{~mm} / \mathrm{y}$ ), but in the absence of false luminal patency, the growth rate of the descending aorta in Fattori and associates' series was only $1.1 \mathrm{~mm} / \mathrm{y}$, which is very close to the rate observed in the current study. Although no details of operative technique are provided by Fattori and associates, their descriptions suggest a much greater prevalence of less-extensive aortic resections, with repairs generally limited to the ascending aorta. This makes it plausible that their higher overall growth rate reflects a higher proportion of patients with the residual false lumen open ( $78 \%$ vs only $43 \%$ in the current series).

Given the importance of residual false luminal patency in determining distal aortic expansion and patient survival, it seems evident that surgical strategy in the treatment of acute type A dissection should include measures not only to optimize hospital survival but also to reduce the likelihood of persistent patency of the distal false lumen. These include avoidance of aortic clamping or resection of the clamp site, use of open distal anastomosis, aggressive resection of the 
intimal tear and adjacent friable aorta, and use of perfusion strategies that direct flow preferentially into the true lumen.

\section{Aortic Reoperations}

We attribute the low rate of proximal reoperation seen in this series to the liberal use of composite aortic root replacement. ${ }^{20}$ It has been our philosophy to resect aortic root tissue rather than to try to reapproximate dissected tissue by using glue or other adjuncts (Table 2). We believe that use of a composite aortic root replacement is a technique that can increasingly safely be used for acute dissection, even in centers not specializing in aortic surgery. ${ }^{1,20}$ However, we also recognize that the most important goal of initial operation in acute type A dissection is to permit immediate survival, with long-term outcome a less-important consideration.

The pattern of distal reoperation that we observed is similar to what has been quoted by others. ${ }^{21}$ The likelihood of reoperation appears to be higher in the presence of Marfan syndrome ( $31 \%$ vs $8 \%$ ), although the numbers were too small to enable formal analysis of risk factors. We speculate that our low incidence of distal reoperation might be a consequence of our determination to resect the intimal tear at the time of the acute dissection because others have correlated failure to resect the intimal tear with risk of late reoperation. ${ }^{13,22}$

\section{Late Mortality}

Overall survival and discharged patients' actuarial survival curves are similar to those of contemporary series from major aortic surgical institutions. ${ }^{22}$ Clearly, patient age is a potent risk factor influencing long-term survival, and its dominance has previously been noted. That the presence of a patent false lumen postoperatively increases late mortality, which depends significantly on aortic pathology, is supported by our data and once more highlights the importance of directing surgical strategy toward closing the false lumen.

The influence of neurological events occurring at the time of the dissection on long-term survival has been described before; indeed, the present study echoes earlier findings that a stroke precipitated by the dissection determines late mortality but not hospital death. ${ }^{23}$ This interesting paradox is perhaps explained by the likelihood that the most severe neurological insults at the time of dissection are accompanied by other related devastating acute processes, such as shock and malperfusion, which then emerge as dominant in the risk factor analysis for hospital death. However, for hospital survivors, the effects of a less-catastrophic stroke might ultimately significantly impair longevity: such patients are likely to have an enhanced risk of bronchopneumonia and other complications associated with chronically impaired levels of physical activity and to be vulnerable to further cerebrovascular events.

The influence of preoperative thrombosis of the false lumen in the ascending aorta on long-term survival remains a matter for speculation. Among patients with a thrombosed false lumen, there seems to be a trend toward less-extensive dissections. This preoperative finding might therefore define a subgroup of patients who have less-aggressive acute type A dissections, perhaps associated with a milder underlying abnormality of the aorta, possibly part of a continuum that includes intramural hematoma, who consequently have a lower risk of subsequent significant distal aortic disease.

Despite our attempts to monitor patients closely after acute type A dissection, the sum of deaths caused by rupture, graft infection, and unknown causes represents $40 \%$ of late deaths, which agrees quite closely with the 2-fold increase in the death rate of this patient group compared with that seen in a healthy matched population. Given the prevalence of aorta-related causes among late deaths and the failure of distal reoperation to have an adverse effect on mortality, there is a strong argument for a continued emphasis on the importance of close surveillance of the distal aorta after acute type A dissection. Nevertheless, 10-year actuarial survival is $66 \%$ versus $79 \%$ for age- and sexmatched control subjects, suggesting that the outlook for patients with a successfully operated acute type A dissection might not be as bleak as previously assumed.

\section{Conclusions}

This study reveals that aortic expansion after type A dissection is typically slow and linear, making distal reoperation relatively uncommon and overall prognosis reasonably favorable. Clearly, some of the factors determining aortic expansion and late survival, including age and sex, preoperative false luminal thrombosis in the ascending aorta, and the presence of new neurological symptoms before surgical intervention, cannot be improved by modifications in surgical technique or by careful follow-up, but our observations also confirm the important role played by residual false luminal flow as a determinant both of aortic dilatation and late survival and thus reinforce the importance of addressing the intimal tear at the time of the operation, which mandates inspection of the aortic arch and its resection where necessary.

\section{References}

1. Halstead JC, Spielvogel D, Meier DM, Rinke S, Bodian C, Malekan R, et al. Composite aortic root replacement in acute type A dissection: time to rethink the indications? Eur J Cardiothorac Surg. 2005;27: 626-32.

2. Dapunt OE, de Asla RA, Griepp EB, Midulla PS, Griepp RB. Computer-generated 3D representations of the aorta: a new tool in the management of aortic aneurysm patients. Thorac Cardiovasc Surg. $1994 ; 42: 25-8$ 
3. Pepe MS. Inferences for events with dependent risks in multiple endpoint studies. J Am Stat Assoc. 1991;86:770-8.

4. Breslow NE, Day NE. Statistical methods in cancer research. Vol II-The design and analysis of cohort studies. Lyon (France): International Agency for Research on Cancer; 1987. p. 91-4, 135-7.

5. Juvonen T, Ergin MA, Galla JD, Lansman SL, Nguyen KH, McCullough JN, et al. Prospective study of the natural history of thoracic aortic aneurysms. Ann Thorac Surg. 1997;63:1533-45.

6. Lai DT, Miller DC, Mitchell RS, Oyer PE, Moore KA, Robbins RC, et al. Acute type A aortic dissection complicated by aortic regurgitation: composite valve graft versus separate valve graft versus conservative valve repair. $J$ Thorac Cardiovasc Surg. 2003;126:1978-86.

7. Fann JI, Smith JA, Miller DC, Mitchell RS, Moore KA, Grunkemeier G, et al. Surgical management of aortic dissection during a 30-year period. Circulation. 1995;92(suppl II):113-21.

8. von Segesser LK, Lorenzetti E, Lachat M, Niederhauser U, Schonbeck M, Vogt PR, et al. Aortic valve preservation in acute type A dissection: is it sound? J Thorac Cardiovasc Surg. 1996;111:381-90.

9. Pansini S, Gagliardotto PV, Pompei E, Parisi F, Bardi G, Castanetto E, et al. Early and late risk factors in surgical treatment of acute type A aortic dissection. Ann Thorac Surg. 1998;66:779-84.

10. Ehrlich M, Fang WC, Grabenwoger M, Cartes-Zumelzu F, Wolner E, Havel M. Perioperative risk factors for mortality in patients with acute type A aortic dissection. Circulation. 1998;98(suppl I):I294-8.

11. Bachet J, Goudot B, Dreyfus GD, Brodaty D, Dubois C, Delentdecker $\mathrm{P}$, et al. Surgery for acute type A aortic dissection: the Hopital Foch experience (1977-1998). Ann Thorac Surg. 1999;67:2006-9.

12. Pessotto R, Santini F, Pugliese P, Montalbano G, Luciani GB, Faggian $\mathrm{G}$, et al. Preservation of the aortic valve in acute type A dissection complicated by aortic regurgitation. Ann Thorac Surg. 1999;67:2010-3.

13. Moon MR, Sundt TM 3rd, Pasque MK, Barner HB, Huddleston CB, Damaino RJ Jr, et al. Does the extent of proximal or distal resection influence outcome for type A dissections? Ann Thorac Surg. 2001;71: 1244-9.

14. Hagan PG, Nienaber CA, Isselbacher EM, Bruckman D, Karavite DJ, Russman PL, et al. The International Registry of Acute Aortic Dissection (IRAD): new insights into an old disease. JAMA. 2000;283: 897-903.

15. Shiono M, Hata M, Sezai A, Negishi N, Sezai Y. Surgical results in acute type A dissection. Ann Thorac Cardiovasc Surg. 2005;11:29-34.

16. Caus T, Frapier JM, Giorgi R, Aymard T, Riberi A, Albat B, et al. Clinical outcome after repair of acute type A dissection in patients over 70 years old. Eur J Cardiothorac Surg. 2002;22:211-7.

17. Neri E, Toscano T, Massetti M, Capaninni G, Carone E, Tucci E, et al. Operation for acute type A dissection in octogenarians: is it justified? J Thorac Cardiovasc Surg. 2001;121:259-67.

18. Sueyoshi E, Sakamoto I, Hayashi K, Yamaguchi T, Imada T. Growth rate of aortic diameter in patients with type $\mathrm{B}$ aortic dissection during the chronic phase. Circulation. 2004;110(suppl II):256-61.

19. Fattori R, Bacchi-Regiani L, Bertaccini P, Napoli G, Fusco F, Longo $\mathrm{M}$, et al. Evolution of aortic dissection after surgical repair. Am J Cardiol. 2000;86:868-872.

20. Ergin MA, McCullough J, Galla JD, Lansman SL, Griepp RB. Radical replacement of the aortic root in acute type A dissection: indications and outcome. Eur J Cardiothorac Surg. 1996;10:840-4.

21. Kirsch M, Soustelle C, Houel R, Hillion ML, Loisance D. Risk factor analysis for proximal and distal reoperations after surgery for acute type A aortic dissection. J Thorac Cardiovasc Surg. 2002;123:318-25.

22. Kazui T, Washiyama N, Bashar AH, Terada H, Suzuki T, Ohkura K, et al. Surgical outcome of acute type A aortic dissection: analysis of risk factors. Ann Thorac Surg. 2002;74:75-81.

23. Pompilio G, Spirito R, Alamanni F, Agrifoglio M, Polvani G, Porqueddu M, et al. Determinants of early and late outcome after surgery for type A aortic dissection. World J Surg. 2001;25:1500-6.

\section{Discussion}

Dr Axel Haverich (Hannover, Germany). Dr Griepp and his group have to be congratulated on an excellent presentation for late outcome in patients with acute type A aortic dissection. As we all know, these patients present with numerous problems at the time of initial presentation; during surgical intervention, which is usually performed on an emergency basis; and during long-term follow-up. The unique features of this abstract are the low operative mortality that was maintained over the years and the true long-term follow-up. This retrospective analysis includes an initial group of 179 patients operated on during a period of 19 years at a single institution. One important difference to other series, including this long-term follow-up, is the fact that more than two thirds of these patients underwent initial replacement of the ascending aorta and portions of the aortic arch. This approach of an open distal anastomosis was pioneered by Dr Griepp and colleagues, and their technique has been adopted by many groups worldwide. As in our own series of late reoperation after initial ascending aorta-only repair in acute type A aortic dissection, many groups have reported on late repair involving the distal ascending aorta or the proximal arch as a frequent indication for late reoperation.

Dr Griepp, do you believe that the initial repair, including an open distal anastomosis, has influenced the considerably lower reoperation rates in your series compared with those of others, and what are your recommendations for the initial repair in the year 2005? Second, in an earlier series from Stanford with an initial 175 patients after repair for acute type A aortic dissection that I had the privilege to publish in Circulation in 1985, there were distinct risk factors for late reoperation. Among these, younger age and the presence of cystic media degeneration clearly triggered a higher incidence for late aortic complications. Why did these risk factors not show up in your analysis, and what are your suggestions in terms of follow-up in this subgroup of patients?

Finally, do your follow-up data, with less than $60 \%$ of patients having repeat CT scans, support your conclusion of a low rate of late aortic expansion or reoperation, and could some late fatal aortic complications be hidden in the large group of late cardiac deaths and deaths from unknown causes?

Thank you for the privilege of discussing this article.

Dr Griepp. Thank you, Dr Haverich, for your kind comments. In response to your first question, I do think that an aggressive resection of the dissected arch probably does have an effect on the long-term prognosis of the remainder of the aorta. That is strictly an impression and a prejudice. I could try and support it by comparing this with other studies, but we all know the difficulties of comparing one institution and one set of studies with another. This study shows a relatively low incidence of distal complications after surgical intervention, and I think the evolution of this field for the next decade is going to be figuring out ways of reducing distal false lumen patency further.

With regard to reoperation, we did not have enough reoperations, our statistician believed, to carry out a true multivariable analysis of determinants of reoperation. Nonetheless, there were a few factors, as you have suggested, that were certainly suggestive.

For instance, there were a small number of patients with Marfan syndrome in this series-I think about $15 \%$ or so-and in that group the overall reoperation rate, not the time-adjusted rate, was $31 \%$, whereas in the rest of the group as a whole, it was only $8 \%$. Therefore quite clearly there are factors here that in larger populations might be risk factors for late reoperation.

It was interesting that when we looked at Marfan syndrome as a predictor of distal expansion of the aorta-more rapid expansion of the aorta-it did not appear. Probably the explanation is that if 
we saw even modest expansion in the patients with Marfan syndrome, they underwent reoperation and were then taken out of the population, in which we are continuing to follow growth rates.

Finally, your third question pounces on the major weakness of the study in that we only had complete follow-up by CT scans in $60 \%$ of the patients, that is, 2 or more CT scans starting at least 6 months after operation. However, we do have quite complete follow-up on survival and reoperation rates.

We did what we believe is a fairly comprehensive analysis of risk factors for late death and expansion in the group for whom we had computed axial tomographic (CAT) scans and those for whom we did not, and the populations did appear to be comparable.

Dr G. Hossein Almassi (Milwaukee, Wis). Dr Griepp, with the rate of enlargement being as large as you presented in your series, what are your recommendations for following up the patients after the repair of a type A dissection? Do you still continue with an annual CT scan forever, or, based on these data, do you recommend to increase the time interval between follow-up scans from annually to maybe every 2 or 3 years?

Dr Griepp. We still had a number of what we think were aorta-related deaths in this population, and some of those, of course, were in patients who did not have CAT scans. I do not think we have the data here to say for sure what the frequency should be, but at the present time our practice is-and I think it is wise to do so-to follow patients annually. Many patients will go along for quite a few years without a change and then will exhibit a change sufficient to trigger reoperation.

The other consideration is that over a period of years, the other side of the risk equation with regard to reoperation changes; that is, risks become lower as techniques improve. Therefore a patient who had a distal aneurysm of $6 \mathrm{~cm} 20$ years ago would not have been operated on, but now we would certainly do it, and I think that will continue to change. Therefore I would suggest annual CAT scans for all of these patients to try and pick out those who will benefit from early reoperation.

Dr Jean Bachet (Paris, France). Thank you very much, Dr Griepp, for this excellent presentation. In my opinion one of the main areas of progress made during the last decade in surgical intervention for acute dissection is the systematic use of the right axillary artery cannulation. In your study have you noted any difference, in terms of late patency of the false lumen, between patients in whom the axillary artery was used directly and patients in whom the femoral artery was used?

My second question relates to the problem of late reoperation on the distal aorta. Concerning the size of the distal aorta, what would you recommend for deciding on a reoperation? It is indeed a difficult issue. Some patients are absolutely asymptomatic, perfectly well, and living a fairly normal life but have a distal aorta of 6 or $7 \mathrm{~cm}$ in diameter, and we know that we have to submit them to the risk of reoperation, paraplegia, death, et cetera. Therefore what is your present policy in such cases?

Dr Griepp. Thank you, Dr Bachet. With regard to the axillary artery, this was used during the last 4 years of the experience. It seems to have had an effect on the patency of the false lumen in the first postoperative scan, but I think this experience is too recent to say whether it will play out over the long-term. We do believe that the antegrade perfusion of the distal dissected aorta is very important, and almost all of these patients, before the era of axillary cannulation, had the cannula inserted into the graft. Therefore we did not perfuse from the femoral artery after the distal anastomosis was done.

With regard to indications for operation in these patients, several years ago we published a multivariate equation for risk of rupture in degenerative aneurysms and atherosclerotic aneurysms of the descending thoracic aorta and then a natural history study in patients with type B dissections. We used the data from those 2 articles to give us some guidance as to what the risk of rupture is, and as you might remember, there are many factors other than just size, having to do with age, the presence of COPD, and so on. Therefore we calculate the annual risk of rupture in these patients using our degenerative aneurysm equation and adding a correction factor for the presence of dissection, which is just simply adding a half a centimeter to the diameter. We then estimate the operative risk, and whichever is less is what we recommend. Obviously a 6-cm aneurysm in a 35-year-old man will almost always require reoperation; in an 85-year-old patient, it probably would not. 


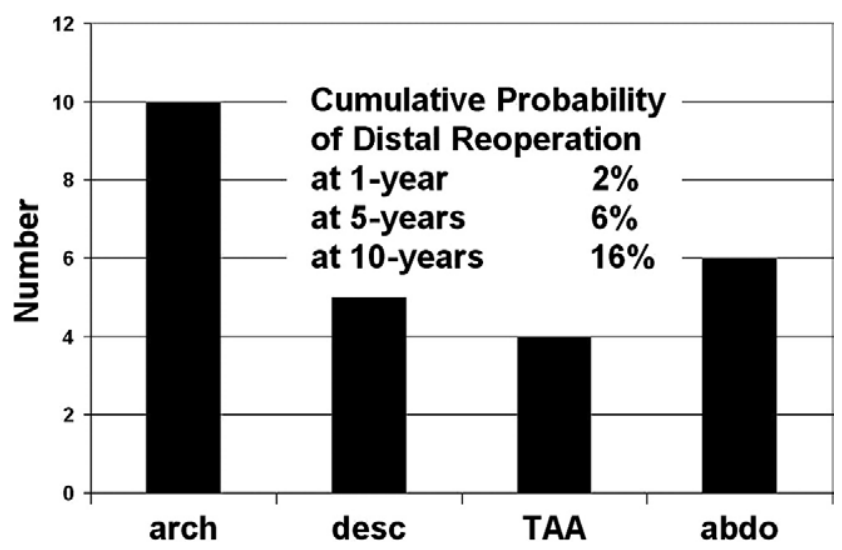

Figure E1. Distribution of distal aortic reoperations by aortic segment replaced. Arch, Resection replacing at least part of the aortic arch;desc, resection limited to the descending thoracic aorta; TAA, thoracoabdominal aneurysm resection;abdo, resection of the infrarenal aorta. Also shown are the cumulative probability functions for requiring a distal reoperation at 1,5 , and 10 years.

TABLE E1. Median aortic segmental growth rates, including the effect of risk factors differing between those patients contributing scans and the group as a whole and of an open false lumen in small aneurysms

\begin{tabular}{|c|c|c|c|}
\hline & $\begin{array}{l}\text { Arch segment } \\
(\mathrm{mm} / \mathrm{y})\end{array}$ & $\begin{array}{l}\text { Descending aortic segment } \\
\qquad(\mathrm{mm} / \mathrm{y})\end{array}$ & $\begin{array}{c}\text { Abdominal segment } \\
\qquad(\mathrm{mm} / \mathrm{y})\end{array}$ \\
\hline Overall & 0.8 & 1.0 & 0.8 \\
\hline Preoperative false lumen patent in the ascending aorta & 0.9 & 1.0 & 0.9 \\
\hline Initial desc size $<4 \mathrm{~cm}$ & & 0.9 & \\
\hline False lumen thrombosed $t$ & & 0.8 & \\
\hline
\end{tabular}

Desc, Descending aortic segment. *Either no flow pattern was observed in the ascending aortic false lumen on echocardiography (usually transthoracic) or no contrast enhancement was seen within the false lumen on computed tomographic scanning. $\uparrow$ The proportion of patients with open and closed false lumen postoperatively did not differ between patients contributing scans and those who did not. 